\title{
Validación de la versión española del cuestionario "Actitudes de los alumnos hacia la integración en educación física (CAIPE-R)"
}

\author{
Carmen OCETE ${ }^{1}$, Javier PÉREZ-TEJERO ${ }^{1}$, Evelia FRANCO ${ }^{2}$ y Javier COTERÓN ${ }^{3}$ \\ ${ }^{1}$ Cátedra Fundación Sanitas de estudios sobre el Deporte Inclusivo. Facultad de Ciencias de la \\ Actividad Física y del Deporte-INEF. Universidad Politécnica de Madrid. \\ ${ }^{2}$ Universidad Pontificia de Comillas. \\ ${ }^{3}$ Facultad de Ciencias de la Actividad Física y del Deporte-INEF. Universidad Politécnica de Madrid.
}

(Recibido, 16 marzo 2017; Aceptado, 14 junio 2017)

RESUMEN: La Educación Física (EF) se muestra, a priori, como un área idónea para trabajar intervenciones educativas para facilitar la inclusión de alumnos con discapacidad, siendo las actitudes de los compañeros sin discapacidad determinantes en este proceso. Este estudio presenta el análisis de validez y fiabilidad de la versión española del cuestionario Children Attitude Integrated Physical Education- Revised (CAIPE-R; Block, 1995). Para ello se utilizó una muestra de 222 alumnos de EF en Educación Secundaria Obligatoria de centros educativos de Madrid, compuesta por 117 varones y 105 mujeres, con edades comprendidas entre $\operatorname{los} 12$ y los 18 años $(\mathrm{M}=14.07 ; \mathrm{SD}=1.31)$. Los resultados muestran la adecuada fiabilidad del cuestionario y la validez del constructo, con un buen ajuste al modelo. La escala adaptada arrojó una reducción a 10 ítems, obteniendo una consistencia interna general por encima de 0.70 , salvo en los ítems 2 y 8 de la escala general y el ítem 9 de la escala específica. El estudio concluye que la versión muestra una validez y fiabilidad adecuadas para detectar las actitudes hacia la inclusion de compañeros con discapacidad en EF en el contexto español.

Palabras clave: Cuestionario, actitud, inclusión, validez, fiabilidad.

Barriers, facilitators and motives of sport participation for athletes with intellectual impairments

\begin{abstract}
Physical Education (PE) seems, a priori, as a suitable area to work educational interventions to facilitate the inclusion of students with disabilities, being peer's attitudes determinant in this process. This study presents the validity and reliability analysis of the "Children Attitude Integrated Physical Education-Revised (CAIPE-R)" questionnaire (Block, 1995) to the Spanish educational context. For this purpose, a sample of 222 PE's students from high school in Madrid was studied, composed of 117 men and 105 women, aged between 12 and 18 years $(\mathrm{M}=14.07$; SD=1.31). The results showed adequate questionnaire reliability and construct validity, with a good fit to the model. The adapted scale showed a reduction to 10 items, obtaining a general internal consistency above 0.70 , except for items 2 and 8 of the general scale and item 9 of the specific scale. The study concluded that this instrument has adequate validity and reliability to detect children's attitudes towards inclusion of peers with disability in PE in the Spanish context.
\end{abstract}

Keywords: Questionnaire, attitude, inclusion, validity, reliability. 
Correspondencia: Carmen Ocete Calvo. Calle Martin Fierro, No $7.3^{\mathrm{a}}$ planta. Facultad de Ciencias de la Actividad Física y del Deporte-INEF. Universidad Politécnica de Madrid. 28040, Madrid. Email: carmen.ocete@upm.es

\section{Introducción}

La actitud se define como un conjunto de creencias cargadas de emoción que predisponen a la persona a actuar de una manera determinada hacia algo concreto, considerándose clave en el cambio de los comportamientos en las personas (Sherrill, 1998). Así, Antonak y Livneh (1988) indican que las actitudes son aprendidas mediante la interacción con otras personas, son complejas y relativamente estables, tienen un objeto social específico como referente, pueden cambiar en cantidad y calidad y son manifestaciones del comportamiento. Siguiendo la Teoría del Comportamiento Planificado (Theory of Planned Behaviour; Ajzen, 1991), las actitudes vienen determinadas por las creencias de los individuos, que se forman a partir de experiencias, el conocimiento y la nueva información adquirida (Kowalski y Rizzo, 1996). Por lo tanto, las actitudes influyen en las intenciones para determinar el comportamiento humano.

En el ámbito educativo, y concretamente en los procesos de inclusión de alumnos con discapacidad en las sesiones de Educación Física (EF), la actitud se muestra como uno de los principales obstáculos que dificultan una inclusión exitosa de estos alumnos, considerándose las actitudes positivas clave para la consecución de dicho éxito (Heikinaro-Johansson, French, Sherrill, y Huuhka, 1995; Hodge, Davis, Woodard, y Sherrill, 2002). La literatura muestra como un factor determinante la actitud de los compañeros sin discapacidad hacia sus compañeros que sí la tienen (Conaster, Block, y Gansneder, 2002; Folsom-Meek y Rizzo, 2002; Hodge, Murata, y Kozub, 2002; Pérez-Tejero, Ocete, Ortega-Vila, y Coterón, 2012).

Desde esta perspectiva, se plantea la necesidad de implementar programas específicos de intervención en el área de EF para conseguir un cambio positivo en la actitud hacia la inclusión, con la consecuente necesidad del análisis de su impacto. Esto pone de relieve la importancia de contar con instrumentos adecuados y verificados para su medición (Rodríguez y Álvarez, 2013).

Surge así la necesidad del uso de herramientas diseñadas específicamente para ser empleadas en el área de EF, siendo la más utilizada en la literatura el cuestionario "Children Attitude Integrated Physical Education-Revised" (CAIPE-R; Block, 1995). Las investigaciones en las que se ha aplicado este instrumento han seguido dos líneas principales de estudio: por un lado, el establecimiento y definición de las características que deben tener las intervenciones para que faciliten un impacto positivo en la actitud de los alumnos sin discapacidad (Archie y Sherrill, 1989; Block y Zeman, 1996; Kalymon, Gettinger, y Hanley-Maxwell, 2010; McManus, Feyes, y Saucier, 2011; Pérez-Tejero y cols.,y cols., 2012; Sherrill, 1998; Slininger, Sherrill, y Jankowski, 2000); y, por otro, el estudio específico del efecto de las intervenciones en el cambio de actitud hacia la inclusión (De Boer, Pijl, Minnaert, y Post, 2014; Krahé y Altwasser, 2006; Liu, Kudláček, y Ondrej, 2010; Reina, López, Jiménez, García-Calvo, y Hutzler, 2011; Slininger y cols.,y cols., 2000; Tripp, French, y Sherrill, 1995).

Además, encontramos numerosos estudios europeos que la han utilizado en la educación primaria, como por ejemplo Jesina, Lucas, Kudlacek, Machova, Janecka, Wittmannova (2006), Yang, Kudlacek y Ondrej, (2010), Panagitou, Kudláček y Evaggelinou (2006), Arampatzi, Mouratidou, Evaggelinou, Koidou, y Barkoukis (2011); así como en los E.E.U.U., como Block y Zeman (1996). Otros ejemplos de autores que lo han administrado también en la educación (c) Psy, Soc, \& Educ, 2017, Vol. 9(3) 
secundaria son Kudláček, Ondřej, y Wittmanová (2011) y Xafopoulos, Kudlacek, y Evaggelinou (2009) y, conjuntamente en primaria y secundaria, Van Biesen, Busciglio y Vanlandewijck (2006), ó Hutzler y Levi (2008).

A pesar de su extendido uso tal y como ha sido expuesto, las propiedades psicométricas del instrumento aún no están completamente probadas (Campos, Ferreira y Block, 2013), y, siendo un tema tan determinante en el ámbito escolar, tampoco se encuentran estudios sobre la adecuación de esta herramienta al contexto español. Por esta razón, en el presente estudio se analizó la validez y fiabilidad del cuestionario CAIPE-R (Block, 1995) a partir de un análisis preliminar de su consistencia interna, así como los posteriores análisis factoriales exploratorio y confirmatorio. Además, se determinó el uso de esta versión española del instrumento para la medición de la actitud hacia la inclusión de alumnos con discapacidad en las sesiones de EF.

\section{Método}

\section{Participantes}

La muestra estuvo formada por 222 sujetos, alumnos de secundaria y bachillerato de 3 centros públicos de la Comunidad de Madrid, siendo 105 mujeres y 117 varones con edades comprendidas entre los 12 y los 16 años $(\mathrm{M}=14.07$; $\mathrm{SD}= \pm 1.31)$.

\section{Instrumento}

La herramienta objeto de análisis es el CAIPE-R (Block, 1995). El cuestionario original está formado por tres apartados: información inicial del alumno (nombre y apellidos, edad, género, nivel de competitividad, familiar o amigo cercano con discapacidad, compañero con discapacidad en clase y compañero con discapacidad en clase de EF); descripción de un hipotético compañero con discapacidad motórica y, por último, relación de 13 ítems pertenecientes a dos escalas: una general que mide la actitud hacia la inclusión en EF (ítems 1-8) y una específica, que mide la actitud hacia la adaptación en los deportes (ítems 9-13); ambas con una escala de tipo Likert de 4 posibilidades $(1=$ sí; $2=$ probablemente sí; $3=$ probablemente no; $4=$ no $)$.

\section{Procedimiento}

Para la traducción del CAIPE-R (Block, 1995) se utilizó el método de back translation con prueba bilingüe y teniendo en cuenta los elementos culturales propios de cada uno de los idiomas (Brislin, 1970; McKay y cols., 1996). Una vez traducido, se administró a un grupo reducido de alumnos $(\mathrm{N}=30)$ para comprobar la comprensión lectora. Se modificaron los aspectos que se detallan a continuación:

- El deporte utilizado, sustituyendo el béisbol por el baloncesto.

- Utilización de dos nombres en castellano para la descripción del compañero hipotético, uno masculino ("Carlos"), y otro femenino ("María").

- Inversión del sentido de los ítems 4 y 6 siendo redactados en positivo.

El cuestionario fue administrado por 3 investigadores especialistas en el área tras obtener la conformidad por parte de la dirección de cada centro. Se realizó durante la sesión de $\mathrm{EF}$, en un tiempo aproximado de 15 minutos. Los padres de los alumnos participantes fueron 
informados a través de una carta, firmando el consentimiento correspondiente de autorización para la participación en el estudio.

\section{Análisis de datos}

Tras el análisis inicial se comprobó las propiedades psicométricas del cuestionario CAIPE-R. En primer lugar se analizó la consistencia interna a través del alfa de Cronbach, utilizando el paquete estadístico SPSS 20.0. Para comprobar que la estructura de los factores con sus ítems se ajustaba adecuadamente al contexto español se realizaron análisis factorial exploratorio y confirmatorio.

\section{Resultados}

Se llevó a cabo un análisis de cada uno de los ítems para valorar la consistencia interna del instrumento adaptado a la población española. El objeto de este análisis fue detectar los ítems que no contribuían a la dimensión evaluada por la escala. Los criterios seguidos para la no eliminación de los ítems fueron:

a) Que la correlación entre el ítem y la escala (excluido el propio ítem) fuera mayor de .30. Se puede observar en los resultados de la Tabla 1, como la correlación de los ítems 2 y 8 pertenecientes a la escala general no llega a este umbral, siendo .18 y .014 respectivamente. En cuanto a la escala específica, los resultados muestran cómo la correlación del ítem 9 tampoco cumple la condición establecida (Tabla 2).

Tabla 1. Correlaciones de los ítems con la escala general (EG) excluido el propio ítem (general).

\begin{tabular}{|c|c|c|c|c|c|c|c|c|}
\hline & $\begin{array}{l}\text { EG sin } \\
\text { ítem } 1\end{array}$ & $\begin{array}{l}\text { EG sin } \\
\text { ítem } 2\end{array}$ & $\begin{array}{l}\text { EG sin } \\
\text { ítem } 3\end{array}$ & $\begin{array}{l}\text { EG sin } \\
\text { ítem } 4\end{array}$ & $\begin{array}{l}\text { EG sin } \\
\text { ítem }\end{array}$ & $\begin{array}{l}\text { EG sin } \\
\text { ítem }\end{array}$ & $\begin{array}{l}\text { EG sin } \\
\text { ítem } 7\end{array}$ & $\begin{array}{l}\text { EG sin } \\
\text { ítem } 8\end{array}$ \\
\hline Ítem 1 & $.54 *$ & & & & & & & \\
\hline Ítem 2 & & .09 & & & & & & \\
\hline Ítem 3 & & & $.58^{*}$ & & & & & \\
\hline Ítem 4 & & & & $.56^{*}$ & & & & \\
\hline Ítem 5 & & & & & $.35^{*}$ & & & \\
\hline İtem 6 & & & & & & $.52^{*}$ & & \\
\hline Ítem 7 & & & & & & & $.60 *$ & \\
\hline Ítem 8 & & & & & & & & .17 \\
\hline
\end{tabular}

* Índice de correlación superior a .30

Tabla 2. Correlaciones de los ítems con la escala específica (EE) excluido el propio ítem.

\begin{tabular}{lcccc}
\hline & EE sin ítem 1 & EE $\sin$ ítem 2 & EE $\sin$ ítem 3 & EE $\sin$ ítem 4 \\
\hline Ítem 9 & -.04 & & & \\
Ítem 10 & & $.54^{*}$ & & \\
Ítem 11 & & & $.60^{*}$ & \\
Ítem 12 & & & & $.50^{*}$ \\
\hline
\end{tabular}

* Índice de correlación superior a .30 
b) Que el coeficiente de alfa de Cronbach de la escala no aumentase al eliminar dicho ítem. Como se observa en la Tabla 3, la fiabilidad de la escala aumenta al eliminar los ítems 2 y 8 de la escala general, y el ítem 9 de la escala específica.

Tabla 3. Alfa de Cronbach de las escalas general y específica al eliminar ítems.

\begin{tabular}{lc|lc}
\hline & Alfa de Cronbach & & $\begin{array}{c}\text { Alfa de } \\
\text { Cronbach }\end{array}$ \\
\hline Escala General & .72 & Escala Específica & .65 \\
Sin ítem Sin item 1 & .66 & Sin ítem 9* & .78 \\
Sin ítem 2* & .74 & Sin ítem 10 & .52 \\
Sin ítem 3 & .65 & Sin ítem 11 & .49 \\
Sin ítem 4 & .65 & Sin ítem 12 & .55 \\
Sin ítem 5 & .71 & Sin ítem 13 & .54 \\
Sin ítem 6 & .67 & & \\
Sin ítem 7 & .66 & & \\
Sin ítem 8* & .73 & & \\
\hline
\end{tabular}

*Ítems cuya eliminación conlleva un aumento del alfa de Cronbach

c) Que el ítem obtuviera una diferencia significativa entre las puntuaciones medias del ítem al comparar los grupos extremos (cortados por el percentil 25 y 75), es decir, suficiente valor discriminativo. En este caso, tanto en la escala general como en la específica, todos los ítems mostraron un aceptable poder discriminativo, siendo el alfa de Cronbach de las escalas resultantes de .74 para la general, y .78 para la específica.

Tabla 4. Diferencia de medias en las puntuaciones de los ítems entre los grupos extremos.

\begin{tabular}{lccc}
\hline Ítems & Grupo percentil 25 M & Grupo percentil 75 M & p \\
\hline Escala General & & & \\
\hline Ítem 1 & $2.69(.05)$ & $4.00(.01)$ & .001 \\
Ítem 2 & $1.77(.05)$ & $3.24(.03)$ & .001 \\
Ítem 3 & $1.77(.05)$ & $4.00(.01)$ & .001 \\
Ítem 4 & $2.61(.06)$ & $4.00(.01)$ & .001 \\
Ítem 5 & $1.00(.01)$ & $3.43(.05)$ & .001 \\
Ítem 6 & $2.78(.06)$ & $4.00(.01)$ & .001 \\
Ítem 7 & $2.73(.06)$ & $4.00(.01)$ & .001 \\
Ítem 8 & $2.58(.06)$ & $4.00(.01)$ & .001 \\
\hline Escala Específica & & & \\
\hline Ítem 9 & $1.63(.05)$ & $3.39(.05)$ & .001 \\
Ítem 10 & $1.64(.06)$ & $4.00(.01)$ & .001 \\
Ítem 11 & $1.55(.06)$ & $4.00(.01)$ & .001 \\
Ítem 12 & $2.60(.06)$ & $4.00(.01)$ & .001 \\
Ítem 13 & $1.49(.06)$ & $4.00(.01)$ & .001 \\
\hline
\end{tabular}




\section{Validez de constructo: análisis factorial confirmatorio}

Para tratar de confirmar la estructura factorial original de la herramienta se llevó a cabo un análisis factorial confirmatorio considerando las dos dimensiones establecidas originalmente (escala general y específica) y los diez indicadores resultantes tras el análisis de ítems realizado previamente. El ajuste del modelo se evaluó de acuerdo a los principales índices de bondad de ajuste resultando satisfactorios en base a los valores generalmente recomendados GFI $>0.90 \mathrm{y}$ RMSEA $<0.08$ (Hair, Black, Babin, Anderson, y Tatham, 2006), de tal manera que los índices fit incrementales son superiores a .90, el RMSR es igual .05 y el RMSEA inferior a .08 (.06).

Tabla 5. Medida de bondad de ajuste del análisis factorial.

\begin{tabular}{cccccc}
\hline Estadísticos de ajuste del modelo obtenidos en el AFC & $\chi^{2} /$ d.f & RMSR & RMSEA & NFI & CFI \\
\hline & 3.54 & .05 & .06 & .93 & .95 \\
\hline
\end{tabular}

La fiabilidad compuesta conjunta de los ítems observados y del modelo proporcionó un valor de .71 para la escala general y de .86 para la escala específica, mientras que la varianza media extractada del modelo fue de .12 para el constructo de actitud general y de .20 para el constructo de actitud específica. Tal y como propone Bollen (1989), se estimó la validez convergente a partir de la magnitud de cargas factoriales. La magnitud de las mismas resultó ser significativa $(\mathrm{t}>1.96)$ en todos los indicadores con valores superiores a .50 en todos los ítems a excepción del ítem 5 (Tabla 6), pudiéndose aceptar la validez de los indicadores (Hair, Black, Babin, Anderson, y Tatham, 2006). La validez discriminante se evaluó mediante la observación de la correlación entre constructos. Dicha correlación resultó significativamente distinta de $1(\mathrm{r}=.31)$.

Tabla 6. Saturaciones de los ítems en cada factor.

\begin{tabular}{lcc}
\hline & \multicolumn{2}{c}{ Factor } \\
\cline { 2 - 3 } & 1 & 2 \\
Item 1 & $\mathbf{. 6 4 0}$ & .055 \\
Item 3 & $\mathbf{. 6 5 0}$ & .036 \\
Ítem 4 & $\mathbf{. 6 4 3}$ & .111 \\
Item 5 & $\mathbf{. 3 8 7}$ & .088 \\
Ítem 6 & $\mathbf{. 5 9 4}$ & .183 \\
Item 7 & $\mathbf{. 7 0 9}$ & .223 \\
Ítem 10 & .039 & $\mathbf{. 7 0 5}$ \\
Ítem 11 & .056 & $\mathbf{. 7 6 9}$ \\
Ítem 12 & .212 & $\mathbf{. 5 9 8}$ \\
Ítem 13 & .005 & $\mathbf{. 6 7 1}$ \\
\hline
\end{tabular}

\section{Validez de constructo: análisis factorial exploratorio}

Los ítems que cumplieron con los criterios establecidos en el apartado anterior fueron sometidos a un análisis factorial. El objetivo fue corroborar la presencia de la estructura factorial original en la adaptación española, por lo que se procedió a realizar un análisis factorial confirmatorio mediante el método de Máxima Verosimilitud (rotación Varimax). El modelo obtenido de dos factores consiguió explicar un 53.95\% de la varianza. Las medidas de 
calidad inicial del análisis resultaron ser muy satisfactorias, con un índice de adecuación muestral de $\mathrm{KMO}=.767$ y una prueba de esfericidad de Bartlett significativa $(\chi 2=640.27$, gl $=45, \mathrm{p}<0.001)$. La matriz de factores rotados (Tabla 7) muestra cómo los ítems 1, 3, 4, 5, 6 y 7 saturan alto en el primer factor (correspondiente a la escala general en la herramienta original), mientras que los ítems 10, 11, 12 y 13 lo hacen en el segundo factor (correspondiente a la escala específica en la herramienta original).

Tabla 7. Carga de los ítems en los factores.

\begin{tabular}{lcc}
\hline & \multicolumn{2}{c}{ Factor } \\
\hline & 1 & 2 \\
Item 1 & $\mathbf{. 6 4 0}$ & .055 \\
Item 3 & $\mathbf{. 6 5 0}$ & .036 \\
Ítem 4 & $\mathbf{. 6 4 3}$ & .111 \\
Item 5 & $\mathbf{. 3 8 7}$ & .088 \\
Ítem 6 & $\mathbf{. 5 9 4}$ & .183 \\
Item 7 & $\mathbf{. 7 0 9}$ & .223 \\
Ítem 10 & .039 & $\mathbf{. 7 0 5}$ \\
Ítem 11 & .056 & $\mathbf{. 7 6 9}$ \\
Ítem 12 & .212 & $\mathbf{. 5 9 8}$ \\
Ítem 13 & .005 & $\mathbf{. 6 7 1}$ \\
\hline
\end{tabular}

\section{Discusión y conclusiones}

Este estudio presenta el análisis de validez y fiabilidad del cuestionario CAIPE-R (Block, 1995), la herramienta más usada en la bibliografía para la evaluación de las actitudes hacia la inclusión en EF, en el contexto educativo español. El análisis de los resultados muestra una consistencia interna aceptable, salvo para los ítems 2,8 y 9 que se mostraban inferiores lo recomendado (.70) (Browne y Cudeck, 1992), siendo por ello eliminados del cuestionario en su versión española final. Estos resultados coinciden con los obtenidos en la revisión de la validación del cuestionario del propio autor (Block, 1995), respecto al ítem 8 (.15) e ítem 9 (.41), mostrando una mejora de la consistencia interna de la ambas subescalas al ser eliminados ambos. Los resultados relativos al alfa de Cronbach mostraron un valor de .74 para la escala general y .78 para la escala específica, coincidiendo solo con los datos mostrados por Campos, Ferreira y Block (2013) en el caso de la primera subescala, con un valor de 0.72, pero difiriendo en la dimensión específica del cuestionario, siendo de 0.48 .

Esta falta de consistencia interna podría ser debida a la propia redacción de los ítems, ya que la respuesta no evidencia un posicionamiento actitudinal, sino más bien una simple constatación de un hecho. Tomando como ejemplo el ítem 9, "María/Carlos podría hacer un pase largo a un compañero situado en el otro lado de la cancha", se observa que la redacción no hace referencia a la actitud hacia la modificación de los deportes, que es lo que se pretende medir, sino la valoración de la eficacia de un gesto técnico determinado. Por otro lado, tampoco permite evidenciar un claro posicionamiento ya que, que el alumno crea que su compañero es capaz de realizar una acción determinada no implica que el alumno este de acuerdo en modificar las reglas y normas del juego para facilitar la participación de éste en las sesiones de EF. En cuanto a los análisis, las cargas factoriales estandarizadas se distribuyeron en dos 
componentes que se correspondían exactamente con los factores establecidos en el original (actitud hacia la inclusión y modificación en los deportes), lo que corrobora la consistencia de los mismos también en esta versión.

En relación al cambio de deporte usado en el cuestionario original (el béisbol), se decidió sustituirlo por el baloncesto para una mayor comprensión de las situaciones expuestas en los ítems, ya que no es un deporte muy conocido y practicado de manera habitual, ni en Europa ni en España en concreto, ni tampoco demasiado usado como contenido de las clases de EF. Esta modificación ya ha sido realizada de igual manera por autores de otros países a la hora de validar el cuestionario en sus propias lenguas, como el hebreo (Hutzler y Levi, 2008) o checo (Kudláček y cols., 2011). Coincidimos con Hutzler y Lezi (2008), tras el análisis de la consistencia interna en la versión israelí del CAIPE-R, en la reducción de los 13 Items originales, en su caso a 11 ítems (siete en la subescala general y cuatro en la subescala específica deportiva; $\mathrm{A}=0.77$ y $\alpha=0.62$ respectivamente), sin embargo en la versión checa (Kudlacek, Ješina y Wittmanova, 2011) el análisis factorial confirmatorio no se calculó.

La decisión de invertir el sentido de los ítems 4 y 6 fue tomada tras comprobar en el estudio piloto las dudas que generaba su redacción entre el alumnado, facilitando así la comprensión por parte del mismo. De esta manera, se realizó un cambio solo en los verbos: en el ítem 4 se cambió "no sería divertida" por "sería divertida", y en el ítem 6 "no hablaría con él/ella y no sería su amigo" por "hablaría con él/ella y sería su amigo". La inversión del sentido de los ítems se realizó con el consenso de los especialistas. El análisis preliminar de sus propiedades psicométricas y el análisis factorial mostraron concordancia con el resto de los ítems.

El cuestionario definitivo (CAIPER-S) consta de 10 ítems de los 13 establecidos en la versión original. Esta reducción mantiene un alto grado de consistencia en la herramienta y permite su aplicación facilitando una nueva herramienta al contexto español que mide la actitud de los alumnos sin discapacidad hacia la inclusión de sus compañeros que sí la tienen en el área de EF. Sería interesante, no obstante, la revisión de la redacción de los ítems eliminados de la versión española para comprobar si los bajos índices de fiabilidad y consistencia son debidos a su formulación, cuestión que se plantea para un estudio futuro. De cara a propuestas futuras, sería interesante el diseño de un cuestionario basado en la TPB (Azjen, 1991), extensión de la Teoría de la Acción Razonada (Fishbein y Ajzen, 1975), en la cual se fundamenta el CAIPE-R.

\section{Referencias}

Ajzen, I. (1991). The Theory of Planned Behavior. Organizational behavior and human decision processes, 50, 179-211.

Antonak, R., y Livneh, H. (Eds.). (1988). The measurement of attitud toward people with disabilities. Springfield: Charles C.Thomas.

Arampatzi, A., Mouratidou, K., Evaggelinou, C., Koidou, E., yy Barkoukis, V. (2011). Social developmental parameters in primary schools: Inclusive settings' and gender differences on pupils' aggressive and social insecure behaviour and their attitudes towards disability. International Journal of Special Education, 26(2), 55.

Archie, V., y Sherrill, C. (1989). Attitudes toward handicapped peers of mainstreamed and nonmainstreamed children in physical education. Perceptual Motor Skills, 69(1), 319-322. 
Block, M. (1995). Development and validation of the Children's Attitudes Toward Integrated Physical Education -- Revised (CAIPE-R) inventory. Adapted Physical Activity Quarterly, 12(1), 60-77.

Block, M., y Zeman, R. (1996). Including Students With Disabilities In Regular Physical Education: Effects on Nondisabled Children. Adapted Physical Activity Quarterly, 13(1), 38-49.

Bollen, K. (Ed.). (1989). Structural equations with latent variables: Wiley.

Brislin, R. W. (1970). Back-Translation for Cross-Cultural Research. Journal of Cross-Cultural Psychology (September), 1 (3), 185-216

Browne, M. W., y Cudeck, R. (1992). Alternative Ways of Assessing Model Fit. Sociological Methods and Research, 21(2), 230.

Campos, M. J., Ferreira, J. P., y Block, M. (2013). An analysis into the structure, validity and reliability of the children's attitudes towards integrated physical education-revised (CAIPE-R). European Journal of Adapted Physical Activity, 6(2), 29-37.

Conaster, P., Block, M. E., y Gansneder, B. (2002). Aquatic instructors' beliefs toward inclusion: the theory of planned behavior. Adapted Physical Activity Quarterly, 19(2), 172-187.

De Boer, A., Pijl, S., Minnaert, A., y Post, W. (2014). Evaluating the Effectiveness of an Intervention Program to Influence Attitudes of Students Towards Peers with Disabilities. Journal of Autism and Developmental Disorders, 44, 572-583.

Fishbein, M., y Ajzen, I. (Eds.). (1975). Belief, Attitude, Intention, and Behavior: An Introduction to Theory and Research. Reading, MA: Addison-Wesley.

Folsom-Meek, S., y Rizzo, T. (2002). Validating the Physical Educators' Attitude Toward Teaching Individuals With Disabilities III (PEATID III) Survey for Future Professionals. Adapted Physical Activity Quarterly, 19(2), 141.

Hair, J., Black, W., Babin, B., Anderson, R., y Tatham, R. (Eds.). (2006). Multivariate data analysis (Vol. 6). Upper Saddle River, NJ: Pearson Prentice Hall.

Heikinaro-Johansson, P., French, R., Sherrill, C., y Huuhka, H. (1995). Adapted physical education consultant service model to facilitate integration. Adapted Physical Activity Quarterly, 12(1), 12-33.

Hodge, S., Davis, R., Woodard, R., y Sherrill, C. (2002). Comparison of Practicum Types in Changing Preservice Teachers' Attitudes and Perceived Competence. Adapted Physical Activity Quarterly, 19(2), 155-171.

Hodge, S., Murata, N., y Kozub, F. (2002). Physical Educators' Judgments About Inclusion: A New Instrument for Preservice Teachers. Adapted Physical Activity Quarterly, 19(4), 435-452.

Hutzler, Y., y Levi, I. (2008). Including children with disability in physical education: general and specific attitudes of high-school students. European Journal of Adapted Physical Activity 1(2), 21-30.

Jesina, O., Lucas, S., Kudláček, M., Machova, I., Janecka, Z., y Wittmannova, J. (2006). Effect of an intervention program on attitude of elementary school children toward inclusion of children with a disability. Paper presented at the 8th European Congress of Adapted Physical Activity, Olomouc, Rep.Checa. 
Kalymon, K., Gettinger, M., y Hanley-Maxwell, C. (2010). Middle school boys' perspectives on social relationships with peers with disabilities. Remedial and Special Education, 31, 305-316.

Kowalski, E., y Rizzo, T. (1996). Factors Influencing Preservice Student Attitudes Toward Individuals With Disabilities. Adapted Physical Activity Quarterly, 13(2), 180-196.

Krahé, B., y Altwasser, C. (2006). Changing negative attitudes towards persons with physical disabilities: an experimental intervention. Journal of Community and Applied Social Psychology, 16(1), 59-69.

Kudláček, M., Ondřej, J., y Wittmanová, J. (2011). Structure of a Questionnaire on Children's Attitudes towards Inclusive Physical Education (CAIPE-CZ). Paper presented at the Gymnica, Palacky University. http://content.ebscohost.com/pdf27_28/pdf/2011/4U6C/01Dec11/78124743.pdf?T=P\&P= AN\&K=781247

Liu, Y., Kudláček, M., y Ondrej, J. (2010). The influence of paralympic choool day on children's attitudes towards people with disabilities. Acta Universitatis Palackianae Olomucensis. Gymnica 40(2), 63-69.

McKay, R. B., Breslow, M. J., Sangster, R. L., Gabbard, S. M., Reynolds, R. W., Nakamoto, J. M., y Tarnai, J. (1996). Translating survey questionnaires: Lessons learned. New direction for evaluation, Summer(70), 93-104

McManus, J., Feyes, K., y Saucier, D. (2011). Contact and knowledge as predictors of attitudes toward individuals with intellectual disabilities. Journal of Social and Personal Relationships 28 (5), 579-590

Panagiotou, A., Kudláček, M., y Evaggelinou, C. (2006). The effect of the implementation of the "paralympic school - day" program on the attitudes of primary school children towards the inclusion of children with disabilities in physical education. The Scientific Journal for Kinanthropology, 7(2), 83-87.

Ocete, C. (2016). Deporte inclusivo en la escuela: diseño y análisis de un programa de intervención para promover la inclusión del alumnado con discapacidad en Educación Física". Tesis doctoral. Facultad de Ciencias de la Actividad Física y el Deporte (INEF). Universidad Politécnica de Madrid.

Pérez-Tejero, J., Ocete, C., Ortega-Vila, G., y Coterón, J. (2012). Diseño y aplicación de un programa de intervención de práctica deportiva inclusiva y su efecto sobre la actitud hacia la discapacidad: El Campus Inclusivo de Baloncesto. RICYDE. Revista Internacional de Ciencias del Deporte, 3(VI), 258-271.

Reina, R., López, V., Jiménez, M., García-Calvo, T., y Hutzler, Y. (2011). Effects of awareness interventions on children's attitudes toward peers with a visual impairment. International Journal of Rehabilitation Research, 34 (3), 243-248.

Rodríguez, A., y Álvarez, E. (2013). Development and validation of a scale to identify attitudes towards disability in Higher Education. Psicothema, 25(3), 370-376.

Sherrill, C. (Ed.). (1998). Adapted physical activity, recreation and sport: crossdisciplinary and lifespan (5th ed.). Dubuke: Mcgraw-Hill.

Slininger, D., Sherrill, C., y Jankowski, C. (2000). Children's Attitudes Toward Peers With Severe Disabilities: Revisiting Contact Theory. Adapted Physical Activity Quarterly, 17(2), 176-196. 
Tripp, A., French, R., y Sherrill, C. (1995). Contact theory and attitudes of children in physical education programs toward peers with disabilities. Adapted Physical Activity Quarterly, 12(4), 323-332.

Van Biesen, D., Busciglio, A., y Vanlandewijck, Y. (2006). Attitudes towards inclusion of children with disabilities: the effect of the implementation of "A Paralympic School Day" on Flemish elementary children. 8th European Conference of Adapted Physical Activity. Retrieved 26 octubre de 2011, from http://www.eufapa.upol.cz/index.php/eucapa/proceedings/eucapa-2006.html

Xafopoulos, G., Kudláček, M., y Evaggelinou, C. (2009). Effect of the intervention program "Paralympic school day" on attitudes of children attending international school towards inclusion of students with disabilities. Paper presented at the Acta Universitatis Palackianae Olomucensis. Gymnica.

\section{Cuestionario de "actitud hacia la integración en educación física" (CAIPER-S).}

Lee atentamente la descripción que tienes a continuación. Después, contesta las siguientes oraciones, pensando en María/Carlos. Deberás de puntuar de 1 a 4, siendo:

$$
4=\text { Sí, } \quad 3=\text { probablemente sí, } \quad 2=\text { probablemente no, } 1=\text { no }
$$

"María/Carlos tiene la misma edad que tú. Sin embargo, no puede caminar, por lo que utiliza una silla de ruedas para desplazarse. A María/Carlos le gusta jugar a los mismos juegos que a ti pero no lo hace muy bien. A pesar de que puede empujar su silla de ruedas, es más lento que tú y se cansa fácilmente. El puede lanzar una pelota, pero no muy lejos. Puede recibir las pelotas que se tiran directamente a él, y él puede votar y pasar la pelota de baloncesto a otro compañero que este lejos en la cancha, pero no puede lanzar una pelota de baloncesto lo suficientemente alto como para encestar una canasta. Como sus piernas no funcionan, no puede patear una pelota".

\section{Cuestionario a cumplimentar por el estudiante:}

\section{Fecha:}

Nombre y apellidos:

Edad:

¿Cómo te consideras?:

¿Tienes un familiar o amigo cercano con discapacidad?:

¿Has tenido un compañero con discapacidad en tu clase?

¿Has tenido un compañero con discapacidad en tu clase de EF?

\section{CURSO/CLASE:}

\section{Género: $\quad$ MUJER $\square \quad$ HOMBRE}

no competitivo $\square \quad$ algo competitivo

muy competitivo

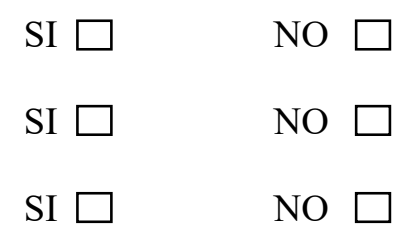




$$
4=\text { Sí, }
$$
3 = probablemente sí,

$2=$ probablemente no, $1=$ no

\begin{tabular}{|l|l|l|l|l|l|}
\hline $\mathrm{N}^{\mathrm{o}}$ & Ítem & \multicolumn{3}{|c|}{ Puntuación } \\
\hline 1 & Estaría bien tener a María/Carlos en clase de Educación Física & 1 & 2 & 3 & 4 \\
\hline 2 & $\begin{array}{l}\text { Si estuviésemos jugando un partido por ejemplo de baloncesto, estaría bien tener a } \\
\text { María/Carlos en mi equipo }\end{array}$ & 1 & 2 & 3 & 4 \\
\hline 3 & $\begin{array}{l}\text { La Educación Física sería divertida si María/Carlos estuviese en mi clase de Educación } \\
\text { Física }\end{array}$ & 1 & 2 & 3 & 4 \\
\hline 4 & $\begin{array}{l}\text { María/Carlos debería tener una Educación Física especial con otros chicos que tuviesen } \\
\text { problemas similares }\end{array}$ & 1 & 2 & 3 & 4 \\
\hline 5 & $\begin{array}{l}\text { Si María/Carlos estuviese en mi clase de Educación Física, hablaría con él/ella y sería } \\
\text { su amigo }\end{array}$ & 1 & 2 & 3 & 4 \\
\hline 6 & $\begin{array}{l}\text { Si María/Carlos estuviese en mi clase de Educación Física, me gustaría ayudarle a } \\
\text { practicar y jugar a juegos }\end{array}$ & 1 & 2 & 3 & 4 \\
\hline 7 & María/Carlos podría tener a alguien para ayudarle a desplazarse por la cancha & 1 & 2 & 3 & 4 \\
\hline 8 & La distancia del tiro a canasta de tres puntos podría estar más cerca para María/Carlos & 1 & 2 & 3 & 4 \\
\hline 9 & Alguien podría ayudar a María/Carlos cuando practica en la cancha de juego & 1 & 2 & 3 & 4 \\
\hline 10 & Las canastas que encesten María/Carlos podrían valer tres puntos & 1 & 2 & 3 & 4 \\
\hline
\end{tabular}

